\title{
Sull'anomalia di Appleton della regione ionosferica $\mathrm{E}^{(*)}$
}

\author{
(About the Appleton anomaly of the ionospheris E region)
}

\author{
P. DoNinicr $(* *)$
}

Ricevuto il 26 Settembre 1972

\begin{abstract}
Sumary. --. The Appleton seasonal anomaly of the ionospheric E region, dedueed both with a constant $\%$, constant. $l$ method and a $(\%, \Omega)$ correlation method from the normalized fol data at Rone, is briefly discussed; in particular, the facts that the amplitude of the anomaly increases with solar astivity and that any explication based upon a "single parameter theory" is unsatisfactory, are emplasized.
\end{abstract}

Risssuxjo. -- L' brevenente illustrata l'anomalia stagionale ali Appleton Alella regione $\mathrm{E}$, dedotta dai dati foE ilellankamento normale ionosferico a Roma sia con un metoslo a $\%$ ell $R$ costanti, sia con un metodo a correlazione $(\%, R)$; sono messi in evidenza l'aumento dell'ampiezza dell'anomalia allaumentare dell'attiviti solare e l'inefficacia di spiegazioni, quali quelle correnti, hasate sulla variazione di un solo parametro frai i molti in gioco.

\section{1. - INTRODUTONE}

Qualche numo fa E. V. Appleton (1) scopri un'interesstante anomalia, che poi da lui la preso nome, nell'andamento anmo della frequenza oritim ordinnrin della regione E della ionosfera, foIs. Precisumente, ̀̀ usuale allermare che per questa regione d̀ ben verificata la teoria di Chapman sulla fotoionizzazione atmosferica al opera di radiazioni solari; norma di tale teoria, per i dati di un determinato Osservatorio ionosferico puó seriversi, in condizioni quasi stazionarie:

$$
\left\{\begin{array}{l}
\text { foE } \mathrm{E}^{4}=\frac{q_{0}}{a^{2} \alpha} \cos \chi=C \cos \chi, \\
a=1,24 \cdot 10^{10} \mathrm{MHz}^{-2} \mathrm{~m}^{-3},
\end{array}\right.
$$

(*) Comunicazione presentata al Convegno annuale dell'Associazione Geofisica Italiana, Roma 26-28 Aprile 1973.

(**) Istituto Nazionale di Geofisica, Roma. 
dove $\chi$ è la distanza zenitale del Sole, qo è il tasso di prorluzione di fotoelettroni per $\chi=0$, a ì il coefficiente cficttivo di ricombinazione. Orbene, se si considerano valori di fol riferentisi a un valore costante della brillanza del sole ionizzante, essi dovrebbero dipendere soltanto da $\chi$ e quindi per un assegnato valore di $\chi$ dlovrebbero essere costanti durante l'auno: si trova invece un massimo invernale.

\section{2. - ANALISI STATISTICA DFI DATI}

A partire dall'andamento normale dei valori mediani mensili di foE ricavati nell'Osservatorio ionosferico di Roma dell'Istituto Nazionale di Geofisica nel periodo 1948-70 e assmendo come indiee della brillanza del Sole ionizzante il numero delle macchie solari $R$, abbiamo estratto i valori mediani mensili di foE $\mathrm{E}^{-1}$ per $R=100, \cos z=0,4 \mathrm{c}$ per $R==100, \cos \chi=0,2$, riportati nelle curve $a, c$ di fig.: il massimo invernale $i$ ben evidente. T'er confronto, sono riportati anche $i$ valori di foE ${ }^{4}$ per $R=0, \cos \%=0,4$, nella curva $b$ : l'andamento è assai meno pronunciato. Questo morlo di rappresentare l'anomalia, che chiameremo " metodo a $\chi$ costante , è sostanzialmente quello seguito da Appleton ( $\left.{ }^{1}\right)$, salvo il fatto che qui la normalizzazione verso assegnati valori di $R$ is stata fatta merliante rette di regressione, mentre là manca una vera $\mathrm{e}$ propria normalizzazione, Appleton avendo considerato medie di valori mediani mensili in un periodo, undecennale, di variazione di $R$.

11 procedimento segruito puó esser criticato perchè mette a confronto dati non omogenei, riferentisi a condizioni variamente diverse dalla stazionarietà (a Roma, $\cos \chi=0,4$ corrisponde circa alle ore 12 a Dicembre, circa alle ore 7 e alle ore 17 a Gingno), cioè a condizioni rappresentate generalmente, jiù che dalla [1], da un'equarione di equilibrio del tipo:

$$
2 a \operatorname{foE} \frac{d(\mathrm{foE})}{d t}=q_{0} \cos \chi-a^{2} \alpha \operatorname{foE}^{4} .
$$

Allo scopo di avere una rappresentazione esente da tale difetto abhiamo ricercato, mese per mese, il legame fra fo $\mathrm{E}^{4} \mathrm{c} \cos \chi$ per $n=0$, mediante rette di regressione in un riferimento logaritmico; come già altri, abbiamo trovato clıe il legame anzirletto può essere espresso dalla relazione:

$$
f_{0} \mathrm{E}^{4}=C_{0}(\cos \chi)^{p}
$$


Come risulta dalla curva $e$ in figura, l'esponente $p$ non presenta una signifieativa variazione stagionale, e ad esso è attribuibile il valor medio $\bar{p}=1,27$ (secondo la teoria di Chapman, e dunque in accorto coll la [1], si sarebbe dovuto avere $\bar{p}=1,00)$; invece, l'andamento della quantita $C_{0}$ (curva $t$ ), che $i$ il valore di foE ${ }^{4}$ per $\chi=0, R=0$, rappresenta l'anomalia di Appleton a Roma, con un regolare, netto massimo invernale.

AFolto recentemente (Gennaio 1973) S. S. Kouris e L. M. Muggleton Lamno comunicato (") i risultati ottenuti applicando ai dati di vari Osservatori ionosferici un low metolo che sostanzialmente ì quello " a correlazione " ora esposto, salvo una maggiore accuratezzi, derivante dal prendere in consinderazione anche la variazione durante l'anno della distanza Terra-Sole, e salvo il fatto che come indice solare è preso, anzicho: $R$, il valore mexlio mensile dell'intensitì della rarlioemissione solare a 2,8 GITL (equivalente, per statisticle a lungo temine, a $I$ ). Relativamente ai dati ali Roma nel periodo 1957-67 essi hanuo ot tenuto per $\bar{p}$ il valore 1,22 e per $C_{0}$ i valori congiunti in fig. con la linea tratteggiata $d^{\prime}$; a parte una sistematica piccola dilierenza, a nostro avviso derivante prineipalmente dalla diversitì dei periodi consislerati, l'accorlo fra gli andamenti $d, d^{\prime}$, è molto buono: ciò costituisce, fra l'altro, una conferma della non influenza della variazione annua della distanza Terra-Sole sulla densità elettronica della regione E.

\section{3. - Discussione Dei Risujatiti}

L'esame delle curve $a \div e$ in figura porta a rilevare $i$ seguenti fatti:

(a) il "metorlo a $\chi$ costante" (curve $a, b, c$ ) si presta bene a mettere in evidenza eventuali dipendenze dell'anomalia da particolari rondizioni: per es., il confronto fra le curve $a, b$, porta a riconoscere una netta dipendenza dell'ampiezza dell'anomalia dall'attivitid solare: tale circostanza non ei pare sia stata sinora rilevata (il metodo seguito da Appleton e poi da molti altri normalizza i dati verso $R$, come accennato, semplicemente mediando su 11 anni; il metorlo di Kouris e Muggleton presenta la stessa limitazione).

(b) Il " metodo a correlazione "(curve $d, d^{\prime}, o$ ) realizza un'efficace perequazione statistica e ben si presta per studi con dati, non sempre omogenei, di vari Osservatori; come aceennato, Kouris e Buggleton lo hamo applicato ai dati di una quarantina di Osservatori ionosferici rlistribuiti in latitudine intorno ai meridiani 50E, 150 $\mathbf{E}$, $75^{\circ} \mathrm{WW}$, otte- 


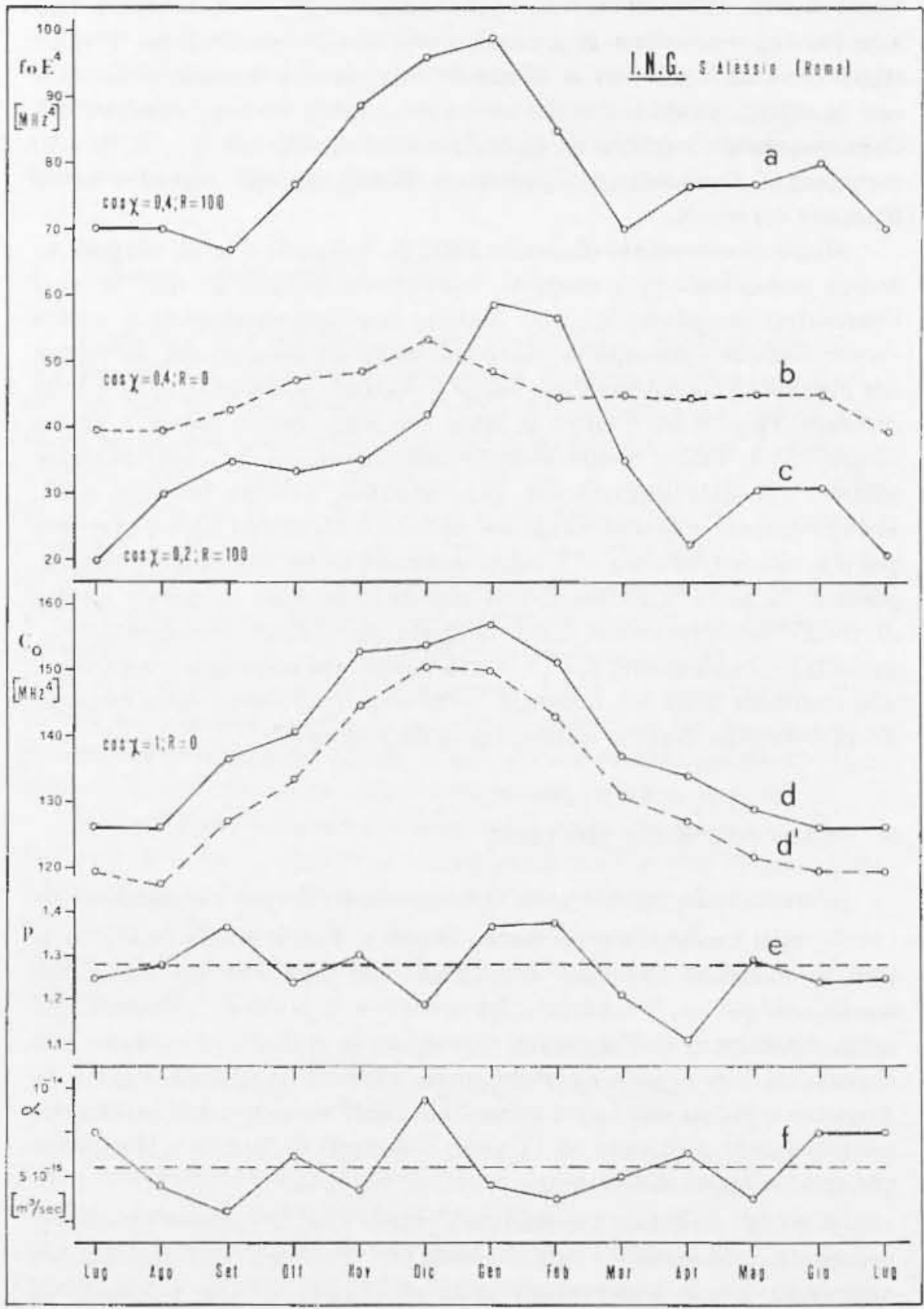


nento $\left(^{2}\right)$ una conforma su scala planetaria delle già note mratteristiche dell'anomalia, principale frat tute il massimo nellinverno locale, con forte effetto di latitudine (ampiezza piceola nella fascia equatoriales, massima a media latitudine, con asimmetrie fra i due emisferi) e aj)prezzabile effeto di longitudine. 1)alla figura scaturiser ma cincostanza abbastanza interessante, e cioc che, come ben risulta dal confronto fra le curve $c\left(\gamma \simeq 78^{\circ}\right)$ ed e, landamento dellespomente $p$ nella [3], al qualo si sarebbe portati a non dare importanza, ì correlato con le pariazioni dell'anomalia a grandi dist:unze zenitali; a posteriori, la cosa non sorprende ove si ponga attenzione al fatto che la penklenza delle rette ali regressione $\log \left(\mathrm{foL}^{4}\right.$, wos $\left.\not\right)$, da chi $p$ is rivato, dipende fortemente dai valori di foE relativi a grandi valori di $\chi$.

Al fine di verificare la signifuativita delle rappresentazioni proposte, abbiamo considerato per ogni mese, e quindi por un determinato valore di qo, eoppie di ore simmetriche rispet to al mezzogiorno locule, quiudl per un determinato valore si cos $\%$; ricavando dai dati normaliz-

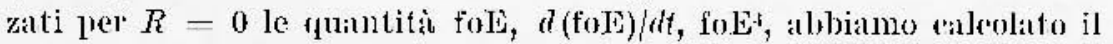
eoefficiente effot tivo di ricombinazione $a$ eliminambo qo eos $\gamma$ fra le coppoie di equazioni [2] rorris]ondenti alle ore scelte; s'è ottemuta la rurva $f$ in figura, che riproduce, contufase come dev'essere, l'andamento dell'ssponente p.

î interessante osiorvare che se si torma a condizioni rigorosamente stazionarie, considerando valori di fol al mezogiono locale, e si ricava qo dalla [1] ntilizzando i predetti valori di a, si ottieno per qo un andamento identico a quello della eurva $f$, con valori intorno a $10^{4} \mathrm{~m}^{3} \mathrm{sec}^{1}$. Tali valori numeriei di qo sono acertabili, mentre l'andamento incompatibile con gli altri risulati. Fa temto presente che dei tre parametri in groco, a cioc foE, a, qo, quello ele certamente conttiene l'anomalia in temini di quantità osservabili ì foL; per a c̀ possibile, come indicato, una valubazone parzialnente indipendesule; per $q_{0}$ ciò che si otliene è soltanto di riversare su esso il carattere di $a$, senza alcun signifieato fisieo. Ciò splegra linanità delle proposte di spiegare l'anomalia di Appleton con teorie a parametro unicom, basate sulla costanza di qo e sull'assunzione cle l'apparente variazione di a sia dovula a corrispondenti variazioni di temperatura e di romposiziono dei gas atmosferici.

Jo stesso si puo dire a proposito della spiegazione, eui inelina attualmente la magrior parte degri studiosi, basala su variazioni di un termine di trasporto verticale collegato al sistema dolle rorrenti Na. In tale teoria, esprimendo, come fatto sinora, la densità eletoroniea mas- 
sima $V_{3}$ in termini di frequenza sitica ordinaria e rlando al termine di trasporto la forma $\operatorname{div}\left(\boldsymbol{N}_{\mathrm{S}} v\right)$, con $v$ velocità verticale di scorrimento, l'equazione di equilibrio puó essere assunta nella forma:

$$
\frac{d(\mathrm{foE})}{d t} \quad \frac{q_{u} \cos \chi-a^{2} \alpha \text { fo } \mathrm{E}^{4}}{2 a \mathrm{fo} \mathrm{E}}-\left[\frac{\text { fo } \mathrm{E}}{2} \frac{\partial t}{\partial h}+v \frac{\partial(\mathrm{foL})}{\partial h}\right] .
$$

Come si vede, il termine fli trasporto viene a rlipenrlere anche da fol e rlal graliente verticale $\partial(\mathrm{foL}) / \partial h$, cioc arl esso viene impressa anctie una variazione ammua che in realtà, anzichí derivare flalla velocità di seorrimento, è propria di folì perchè, per es., deriva da $a$ (che invece, a.l pari di qo, è assunto costante). Sel citato articolo di Kouris e Muggleton (2) ¿̀ fatta un'eccellente rassegna dei lavori basati sulla sjoiegazione in discussione, e la conclusione, come era da aspettarsi, c̀ che gli argomenti a favore sono soltanto qualitativi.

\section{4. - Coyolusioni}

A questo punto, quel che si puó clire è che l'anomalia di Appleton rivela una sino a qualche tempo fa insospettata natura complessa della regione E, contribuendo, con altri fatti quali la riconosciuta non linearità di responso all'attività solare e l'esistenza di una consistente I notturna, ad apjurentare questa regione alla sovrastante regione $\mathrm{F}$ : si viene cosi a costituire un'identità morfologica fra le due regioni (cui probabilmente partecipa anche la regione $\mathbf{D}$ ) in virtù della sua ben nota " anomalia invernale "), Ia (ifferenza fra esse consistendo nella diversa. evicienza del comjortamento "non alla Chapman ". E dunque, jer dar conto dell'anomalia della regione $\mathrm{E}$, posto che sjiegazioni semplici riescono non plausibili, oceorrerà trasferire alla regione $\mathrm{E}$ i meconismi, rli trasjorto e no, projosti e in via di studio per dar conto delle assai più vistose amomalie della regione $\mathrm{F}$.

\section{BIBLIOGRAFIA}

(1) Appleton k. V., 1963. - Jour. Atm. Terr. Phys., 25, 577.

(2) Kouris S. S., M[UGGL.tox T. MI., 1973. - Jour. Atm. Terr. Phys., 35, 141. 